John Carroll University

Carroll Collected

Repeatability of foraging behavior following a simulated predation attempt depends on color morph, sex, and foraging metric in Redbacked salamanders (Plethodon cinereus)

Brian P. Waldron*

Marissa C. Ganzfried*

Cari-Ann M. Hickerson

Carl D. Anthony

Follow this and additional works at: https://collected.jcu.edu/fac_bib_2021

Part of the Biology Commons 


\title{
Repeatability of foraging behavior following a simulated predation attempt depends on color morph, sex, and foraging metric in Red-backed salamanders (Plethodon cinereus)
}

\author{
Brian P. Waldron ${ }^{*, \#}$, Marissa C. Ganzfried, Cari-Ann M. Hickerson \\ and CARL D. ANTHONY
}

Department of Biology, John Carroll University, 1 John Carroll Blvd, University Heights, OH 44118, USAO January 2021, accepted 3 June 2021

\begin{abstract}
Behavioral repeatability greatly affects the capacity of an individual to respond to varying environments. When multiple behaviors within individuals are repeatable and correlated across time or across contexts, it is termed a behavioral syndrome. However, not all behaviors exhibit the same level of repeatability, and relatively few studies have examined repeatability in amphibians. We examined the repeatability of foraging behavior in the Eastern Red-backed salamander (Plethodon cinereus), a color-polymorphic terrestrial salamander, following a simulated predation attempt. We tested several hypotheses: (1) Simulated predation would negatively affect foraging, increasing latency to feed and decreasing the number of prey items eaten in a fixed time period compared to a control group; (2) Because striped color morphs of $P$. cinereus are more aggressive, striped individuals would exhibit "bold" behavior by resuming foraging sooner and consuming more prey; and (3) Foraging behavior would be more repeatable for males. We found that the predation treatment inhibited foraging behavior, although neither morphs nor sexes differed in either forging metric. The number of prey eaten was repeatable for all groups of salamanders. Latency to feed, however, was not repeatable for control salamanders. Simulated predation induced repeatable latencies, but when morphs and sexes were analyzed separately, only unstriped and male salamanders were repeatable, suggesting characteristics of these groups related to behavioral syndromes drive this response. We speculate that the greater repeatability of the unstriped morph's latency to feed may result from more frequent encounters with predators in the leaf litter matrix while foraging. Striped salamanders from the source population, in turn, exhibit greater territorial success, and thus may experience more variation in encounters with predators and conspecifics over the course of their lifespans. Our results illustrate the need to carefully define the behavior and subset of the population to be tested when studying behavioral repeatability or behavioral syndromes.
\end{abstract}


KEY WORDS: repeatability, foraging behavior, behavioral syndrome, animal personality, intraclass correlation coefficient, Eastern Red-backed salamander, Plethodon.

\section{INTRODUCTION}

A behavioral syndrome is a suite of correlated behaviors that remain consistent across contexts (Sih et al. 2004). If, for example, an individual is consistently observed as aggressive towards conspecifics and is also fast to explore a novel environment, the individual would exhibit traits of a "bold/aggressive" behavioral syndrome, whereas an individual submissive to conspecifics and reluctant to explore a novel environment would be deemed "shy/submissive" (Sih et al. 2004). Behavioral syndromes are of particular interest for evolutionary biologists because they can have profound fitness consequences for individuals (Smith \& Blumstein 2008). A behavioral tendency such as boldness that is favorable in an environment with abundant prey and few predators may be detrimental to fitness if predator concentrations were to increase (Sih et al. 2004, 2012; Ballew et al. 2017).

Predation risk can greatly affect optimal foraging behavior, but variation in individuals' responses to predation will depend on the consistency of behavior over time and across contexts. Related to the concept of behavioral syndromes, "animal personality" refers to repeatable differences in a given behavior among individuals, across time and across contexts (Réale et al. 2007). Zebrafish, for example, vary in their latency to feed in the presence or absence of predators and of conspecifics, exhibiting the lowest latency (i.e. bolder behavior) when both predators and conspecifics were present. Behaviors were repeatable when measured across combinations of predator and conspecific presence and absence (Roy et al. 2017). However, in other groups, behavioral repeatability increases with predation risk. Mosquitofish in intermediate turbidity levels - the amount of sediment in the water, which affects visual ability - exhibited repeatable activity and exploratory behavior when exposed to predator chemical cues, whereas behaviors were generally not repeatable when the predator cue was absent (Ehlman et al. 2019). Intermediate turbidity in aquatic systems may increase the predation risk of fish by decreasing their visual ability, whereas clearer waters allow fish to regularly assess threat levels, and high turbidity functions as a refuge for prey from visual predators (Ehlman et al. 2019). Predation risk may therefore induce repeatable behavior when risk is highest because strategies for survival in these contexts may be limited. Alternatively, different individuals in a population may vary in suites of correlated behaviors such that certain members of the population exhibit inflexible bold responses to predation risk, while other members of the population may be more flexible, with variation affected by prior experiences with predators (Mazza et al. 2019).

The presence of behavioral syndromes depends upon the repeatability of each behavior (Brodie \& Russell 1999), as only behaviors that are consistent through time could be reliably correlated across contexts (Bell et al. 2009). Measuring the repeatability of a behavior is a first step in determining whether it could be part of an overarching syndrome. Behavioral repeatability, however, is known to vary between sexes and age classes within a species (Brodie \& Russell 1999; Bell et al. 2009). For instance, males may tend to be more repeatable than females for some behaviors such as foraging (Jenkins 2011), sociability (Strickland et al. 2018) or exploration (Schuett 
\& Dall 2009; Schuett et al. 2010), but this pattern can vary across species and behaviors (Bell et al. 2009; Schuett et al. 2010). Identifying which aspects of behavior are repeatable within a population is valuable in understanding how ecological variation influences the fitness of different segments of the population.

Relevant to the "bold/aggressive" versus "shy/submissive" behavioral syndromes, two color morphs of the Eastern Red-backed salamander (Plethodon cinereus), the striped and unstriped morphs (Fig. 1), have been well studied in the context of ecological divergence. The striped morph has been found to be more territorial and aggressive in territorial contests than the unstriped morph (Reiter et al. 2014). In at least one population, the striped morph had a diet composition of more profitable prey (Anthony et al. 2008), and was found in territories with higher prey abundance (Paluh et al. 2015; Anthony et al. 2017). Observation of opposite sex pairs also revealed striped males with larger females more frequently than were unstriped males (Anthony et al. 2008; Acord et al. 2013). Additionally, Venesky and Anthony (2007) found evidence from museum collections of fewer instances of tail breakage in the striped morph, and following exposure to the scent of a predator, the morph is more likely to display an aggressive posture ("all trunk raised") and remain immobile for longer compared to the unstriped morph. Physiologically, baseline stress levels in the striped morph are lower than that of the unstriped morph (Davis \& Milanovich 2010). These behaviors and tendencies that are more pronounced in the striped morph are indicative of an overall bold/aggressive behavioral type (Sih et al. 2012).

Relatively few studies have examined repeatability of behavior in amphibians (Kelleher et al. 2018), and even fewer have addressed the repeatability of behavior in $P$. cinereus, an otherwise model organism in behavioral studies (Jaeger et al. 2016). One study examined individuals of $P$. cinereus from both edge and interior forest stands, and animals from both habitats were found to exhibit repeatability in multiple aspects of movement behavior in lab trials (Cosentino \& Droney 2016). Additionally, Gibbons et al. (2005) addressed the roles of heritability and learning in foraging of the

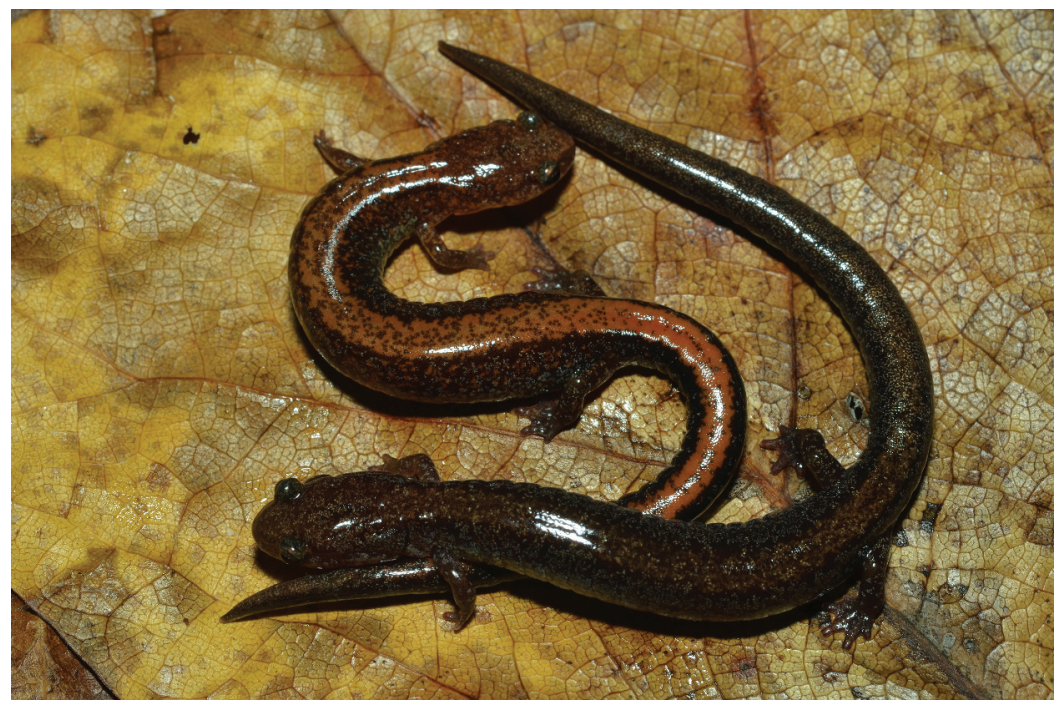

Fig. 1. - Striped and unstriped Eastern Red-backed salamanders, Plethodon cinereus, from Summit County, Ohio, USA. 
salamanders over three life stages with three repeated trials within each stage. Individuals became more efficient in capturing prey after the first trial within a life stage, and some components of foraging behavior were heritable, suggesting a genetic component to differences in foraging behavior among individuals. These results provide evidence that both learning and heritability influence variation in foraging behavior, but it is not yet understood how either morphs or sexes might differ in repeatability, or how factors such as predation attempts experienced while foraging would influence individual behavior.

In the present study, we examined the foraging responses of striped and unstriped salamanders following a simulated predation attempt (hereafter, "simulated predation") to determine (1) if color morphs or sexes differ in their response to simulated predation, and (2) if the responses are repeatable through time. Both males and females exhibit territorial and aggressive behavior (Jaeger 1984), but there is some evidence to suggest that males have a lower latency to move in a novel environment (Cosentino et al. 2017). We predicted that male salamanders and the more aggressive striped morph would have a shorter latency to feed and higher foraging efficiency following simulated predation, and that foraging metrics would be more repeatable for males compared to females.

\section{MATERIALS AND METHODS}

\section{Collection site and experimental design}

On 22 September 2015, 40 striped and 40 unstriped adult individuals of $P$. cinereus were collected from a forested hillside in northern Summit County, Ohio. Within each color morph, we collected equal numbers of each sex (adult males > $32 \mathrm{~mm}$ and adult females > $34 \mathrm{~mm}$ SVL; Anthony \& Pfingsten 2013). Salamanders were held in the lab individually in $470 \mathrm{~mL}$ Pyrex dishes (interior dimensions $5 \mathrm{~cm}$ high by $10.5 \mathrm{~cm}$ in diameter) at a temperature range maintained between $19.0-21.5{ }^{\circ} \mathrm{C}$ and a natural photoperiod (11 Light:13 Dark) for the duration of the study. Moist paper towel substrates were replaced once per week the day after each weekly trial. To ensure that salamander had similar hunger levels before the trials, each salamander was given 25 wingless fruit flies 1 week following collection, approximately 1 week before trials began. Equal numbers of salamanders of each combination of morph and sex (10 striped males, 10 unstriped males, 10 striped females, and 10 unstriped females) were randomly assigned to one of two groups: Control with no treatment, or simulated predation in which during each trial, the salamander was seized mid-body with pressure-sensitive forceps for $5 \mathrm{sec}$ (i.e. "attacked") immediately before commencing the feeding trials. We conducted trials 3 days per week between 11:00 and 15:00 hr, with approximately equal numbers of each morph, sex, and treatment group each day. Each salamander thus underwent a feeding trial once per week, and salamanders fed only on their respective trial day. To assess repeatability of behavior, we tested each salamander a total of 3 times, once per week for 3 weeks ( 240 trials in total).

For feeding trials, we placed salamanders in testing chambers $(15 \mathrm{~cm}$ diameter Petri dishes with moist filter paper substrate) $24 \mathrm{hr}$ before each trial. After this acclimation, we either simulated the predation attempt or performed no treatment. Immediately thereafter, 15 live wingless fruit flies were dropped into the chamber. We recorded the amount of time in seconds that an individual took to successfully consume the first fly (i.e. latency) as well as the number of flies consumed at the end of $10 \mathrm{~min}$. In a small number of trials, between one and four flies stuck to their container, and we did not attempt to remove them so as to minimize the time between dispensing flies and starting the trial. In these cases, the raw number of flies eaten was corrected by dividing by the number dispensed, multiplying by 15 , and rounding to the nearest whole number. If an individual did not eat any flies in the 10 -min period (1.7\% of trials), the latency score is reported as $600 \mathrm{sec}$, a conservative approach as latency could extend well beyond the 
trial period in these cases. Any flies remaining in the dish at the end of the trial were placed along with the salamander into the Pyrex housing dish and discarded if uneaten the following day.

\section{Data analysis}

Prior to analyses, latency to feed was $\log _{2}$ transformed to meet assumptions of normality. Response variables were analyzed separately for all statistical analyses. We first tested for effects of treatment, morph, and sex on the response variable while accounting for repeated measures using generalized linear mixed-effects models (GLMM) in the 'Ime4' package in R 3.5.3 (Bates et al. 2015; $\mathrm{R}$ Core Team 2019). We used a Gaussian error distribution for latency, and a binomial error distribution with a logit link for the proportion of flies eaten. Treatment, morph, and sex, as well as all possible interactions were designated as fixed effects. Trial number (i.e. week) and salamander identity were included as separate random effects with random intercepts. We used the R package "emmeans" (Lenth et al. 2019) to estimate the marginal means for significant fixed effects.

Behavioral repeatability estimates were calculated as $R$, the intraclass correlation coefficient (ICC; Sokal \& Rohlf 1981), in which values typically range from 0 to 1: where 1 indicates that all observed variability resulted from differences between individuals (completely repeatable), and a value of 0 indicates that all variance results from variation within individuals (not at all repeatable). ICC values were calculated using GLMM-based repeatabilities where the between-individual and within individual variance components can be extracted directly from the GLMM (Nakagawa \& Schielzeth 2010). Latency was analyzed as Gaussian data, while the number of flies eaten out of 15 was analyzed as binomial proportion data with a logit link. We sought to determine if behaviors were repeatable within each treatment group, and also whether repeatability differed for sexes and color morphs. For each response variable, within both the control and treatment groups, we used the R package "rptR" (Stoffel et al. 2017) to perform five GLMMs, with salamander identity as the random effect: one GLMM included all individuals in the treatment group ("overall"; $N=40$ ), and the remaining four models respectively included only the striped, unstriped, male, and female individuals within that treatment group ( $N=20$ for each GLMM). For example, the GLMM for control-group striped individuals included all striped individuals in the control group regardless of sex (i.e. $N=10$ striped males and $N=10$ striped females), and the GLMM for control-group males included all male salamanders in the group regardless of color morph $(N=10$ striped males and $N=10$ unstriped males). To account for the effect of color morph in calculations of $R$ for either sex, and to account for the effect of sex in calculations for either morph, we included that variable as a fixed effect in the GLMM. The GLMM including all individuals in the treatment group ("overall") included fixed effects of both morph and sex. Our repeatability estimates thus represent adjusted repeatability (Nakagawa \& Schielzeth 2010). For the proportion of flies eaten, we report repeatability estimates on both the latent (link) scale and the original scale, but we focus interpretations on the link-scale results (Nakagawa \& Schielzeth 2010). Confidence intervals were calculated using 1000 parametric bootstrap replicates. We used likelihood-ratio tests (LRT) to assess statistical significance of repeatability, adjusting $P$-values by applying a Benjamini-Hochberg correction for multiple tests (20 models in total).

Finally, for the proportion of flies eaten, we also report repeatability values based upon arcsine-transformed raw data, analyzed as Gaussian data in a GLMM in "rptR". Although we focus interpretations on the proportion data analyzed with a binomial GLMM as detailed above, the arcsine transformation has been widely used for proportion data (Nakagawa \& Schielzeth 2010), and we present these results to explore how repeatability estimates and assessments of statistical significance vary with either method. 
Morph, sex, and treatment effects

When considering all weeks, control salamanders on average ate 12.1 flies $(\mathrm{SD}=2.7)$ of the 15 flies provided and took $27.7 \mathrm{sec}(\mathrm{SD}=46.7)$ to consume the first fly. Attacked salamanders ate 10.3 flies $(\mathrm{SD}=3.7)$, and took $67.9 \mathrm{sec}(\mathrm{SD}=99.2)$ to feed. Simulated predation inhibited feeding most drastically in week 1, in which salamanders on average ate 9.2 flies $(\mathrm{SD}=4.4)$ and took $111.8 \mathrm{sec}(\mathrm{SD}=159.7)$ to feed, compared to 10.8 flies $(S D=3.4)$ and $46.0 \mathrm{sec}(\mathrm{SD}=69.0)$ to feed across weeks 2 and 3. Control salamanders exhibited consistent foraging behavior in week 1 (flies $=11.9, \mathrm{SD}=2.9$; latency $=30.4 \mathrm{sec}, \mathrm{SD}=43.3$ ) relative to weeks 2 and 3 (flies = 12.2, $\mathrm{SD}=2.6$; latency $=26.4 \mathrm{sec}, \mathrm{SD}=48.5$; Fig. 2 ).

In our two GLMM models (one for each response variable) including morph, sex, treatment, and their interaction terms as fixed effects, only treatment (control vs simulated predation) had a significant effect on the proportion of flies eaten (Wald chisquare $=4.03, \mathrm{df}=1, P=0.045)$ and latency to feed $\left(F_{(1,72)}=18.09, P<0.001\right)$. No interactions were significant. Based on estimated marginal means, control and
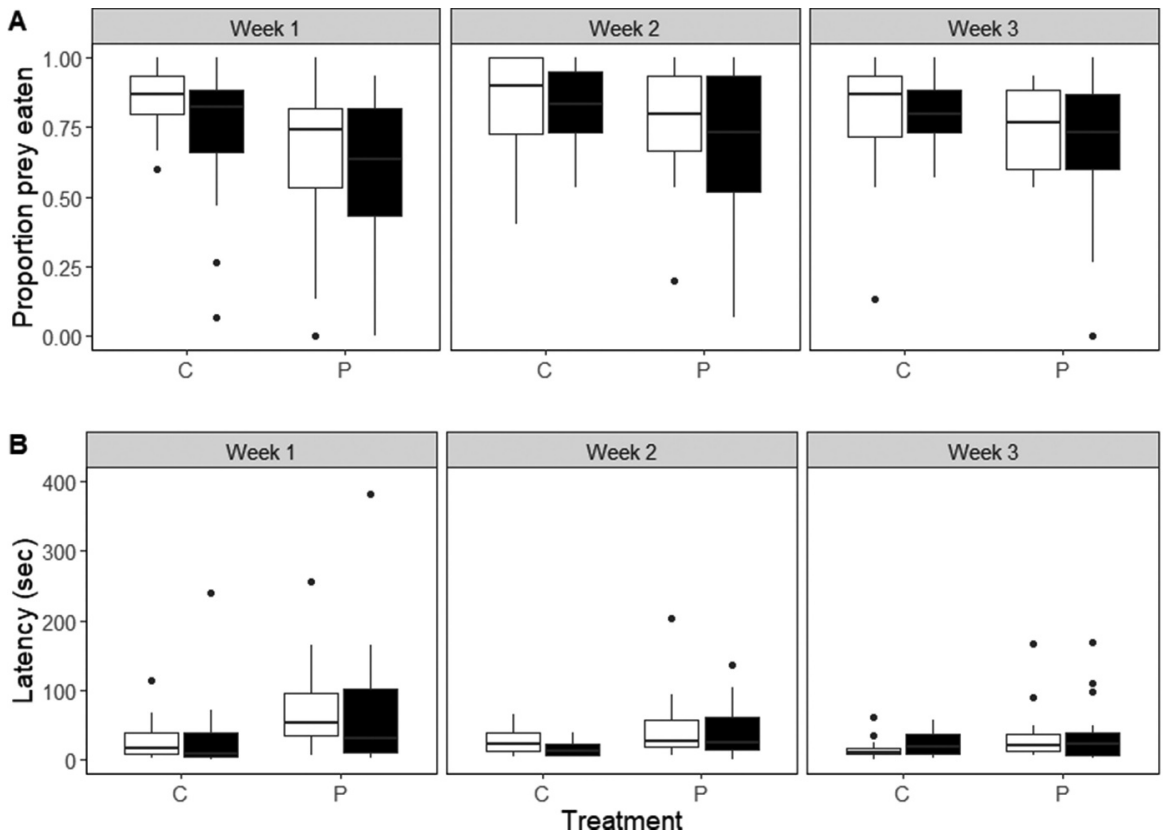

Morph
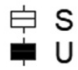

Fig. 2. - Foraging behavior of $P$. cinereus across 3 weeks in a control group (Treatment $=\mathrm{C} ; N=40)$ and a group subjected to a simulated predation attempt immediately before the feeding trial (Treatment $=\mathrm{P}$; $N=40$ ). Equal numbers of each color morph were in each treatment group ( $\mathrm{S}=\mathrm{Striped}$; $\mathrm{U}=\mathrm{Unstriped})$. (A) Proportion of the prey eaten (maximum of 15 flies) within $10 \mathrm{~min}$; (B) Untransformed latency to first feed in seconds after flies were fully dispensed into the testing chamber. To improve readability, five data points with latencies > $500 \mathrm{sec}$ are not visible: one striped and two unstriped salamanders in the week 1 predation group $(600 \mathrm{sec})$, one unstriped salamander in the week 3 predation group $(600 \mathrm{sec})$, and one striped salamander in the week 3 control group (517 sec). 
attacked salamanders ate $12.6(95 \% \mathrm{CI}=11.8-13.3)$ and $10.8(95 \% \mathrm{CI}=9.5-11.8)$ flies and had latencies of $14.7 \mathrm{sec}(95 \% \mathrm{CI}=10.4-21.0)$ and $31.8 \mathrm{sec}(95 \% \mathrm{CI}=22.5-44.9)$, respectively. That is, simulated predation decreased the proportion of flies eaten by $14.3 \%$ and increased latency by $116.3 \%$ relative to the control group.

\section{Repeatability}

The proportion of flies eaten based on the binomial GLMM was repeatable in all cases within both the control and attacked groups, and all values were statistically significant based on LRT after correction for multiple comparisons. These ICC values ranged from 0.08 to 0.17 (Table 1). Results were nearly identical on the link and original scales. Latency to feed, however, was not significantly repeatable for the control group. Latency was repeatable for the attacked group when all individuals were included $(R=0.26)$, but when morphs and sexes were analyzed individually, only unstriped $(R=0.32)$ and male salamanders $(R=0.37)$ exhibited significantly repeatable behavior (Table 1).

Arcsine-transformed data for the proportion of flies eaten yielded considerably higher repeatability estimates than in the binomial GLMM, ranging from 0.31 to 0.48 (Table 2). However, all values were statistically significant, suggesting that presence of repeatable prey consumption would still be detected for all groups given our data set.

\section{DISCUSSION}

Consistent with our predictions, a simulated predation attempt inhibited foraging behavior in Plethodon cinereus. Although we did not find effects of color morph or sex on either foraging metric, patterns within individuals across repeated trials behavioral repeatability - differed depending on the foraging metric, treatment, morph, and sex. For the number of flies eaten in $10 \mathrm{~min}$, we found that behavior was repeatable regardless of any other variable. Latency to feed, on the other hand, was not repeatable for the control group. Stochasticity in the control salamanders' encounters with moving prey items likely explains variation in latencies to feed, despite the number of prey items eaten following the latency period being repeatable for the same individuals. Interestingly, the simulated predation attempt appears to induce a repeatable latency response in which individuals are consistent in how quickly they will begin foraging following a stressful event. When explored further, this effect appears to be driven largely by unstriped and male salamanders. These results demonstrate that although color morphs and sexes may not differ on average for a behavior (e.g. latency to feed following stress), repeatability of individual behavior over time can vary across groups.

For studies of behavioral syndromes, selecting a metric to act as a behavioral proxy is a critical decision. Latency to explore a novel environment and latency to feed following a threat stimulus can provide different results, despite both being methods to assess boldness (Kelleher et al. 2018). We show that within the same behavioral assay (i.e. simulated predation) different interpretations can be achieved with different metrics - in our study, the latency to feed versus the subsequent rate of foraging. Not surprisingly, simulated predation increased the latency to feed, and decreased the number of flies eaten in a fixed period of time. However, the reduction in flies eaten 
Table 1.

Repeatability, calculated as the intraclass correlation coefficients (ICC), $R$, of two foraging metrics across three repeated trials.

\begin{tabular}{|c|c|c|c|c|c|c|c|}
\hline \multicolumn{2}{|c|}{ Flies eaten: Control } & \multicolumn{2}{|c|}{ Link scale } & \multirow[b]{2}{*}{$P$} & \multicolumn{2}{|c|}{ Original scale } & \multirow[b]{2}{*}{$P$} \\
\hline & $N$ & $R$ & $95 \% \mathrm{CI}$ & & $R$ & $95 \% \mathrm{CI}$ & \\
\hline Overall & 40 & 0.09 & $0.03-0.14$ & 0.000 & 0.10 & $0.03-0.16$ & 0.000 \\
\hline Striped & 20 & 0.09 & $0.01-0.17$ & 0.001 & 0.09 & $0.01-0.19$ & 0.001 \\
\hline Unstriped & 20 & 0.09 & $0.01-0.18$ & 0.002 & 0.10 & $0.01-0.19$ & 0.002 \\
\hline Male & 20 & 0.10 & $0.01-0.19$ & 0.000 & 0.11 & $0.01-0.22$ & 0.000 \\
\hline Female & 20 & 0.08 & $0.00-0.16$ & 0.003 & 0.08 & $0.00-0.18$ & 0.003 \\
\hline \multicolumn{2}{|c|}{ Flies eaten: Predation } & & & & $R$ & $95 \% \mathrm{CI}$ & $P$ \\
\hline Overall & 40 & 0.14 & $0.04-0.22$ & 0.000 & 0.16 & $0.05-0.27$ & 0.000 \\
\hline Striped & 20 & 0.09 & $0.00-0.18$ & 0.004 & 0.09 & $0.00-0.18$ & 0.004 \\
\hline Unstriped & 20 & 0.17 & $0.03-0.28$ & 0.000 & 0.19 & $0.03-0.35$ & 0.000 \\
\hline Male & 20 & 0.08 & $0.00-0.16$ & 0.008 & 0.09 & $0.00-0.19$ & 0.008 \\
\hline Female & 20 & 0.17 & $0.02-0.29$ & 0.000 & 0.19 & $0.02-0.34$ & 0.000 \\
\hline \multicolumn{8}{|c|}{ Latency: Control } \\
\hline Overall & 40 & 0.16 & $0.00-0.37$ & 0.085 & & & \\
\hline Striped & 20 & 0.28 & $0.00-0.53$ & 0.041 & & & \\
\hline Unstriped & 20 & 0.06 & $0.00-0.34$ & 0.417 & & & \\
\hline Male & 20 & 0.23 & $0.00-0.48$ & 0.058 & & & \\
\hline Female & 20 & 0.08 & $0.00-0.37$ & 0.317 & & & \\
\hline \multicolumn{8}{|c|}{ Latency: Predation } \\
\hline Overall & 40 & 0.26 & $0.06-0.46$ & 0.007 & & & \\
\hline Striped & 20 & 0.17 & $0.00-0.45$ & 0.161 & & & \\
\hline Unstriped & 20 & 0.32 & $0.03-0.59$ & 0.019 & & & \\
\hline Male & 20 & 0.37 & $0.06-0.63$ & 0.007 & & & \\
\hline Female & 20 & 0.11 & $0.00-0.39$ & 0.289 & & & \\
\hline
\end{tabular}

Control and simulated predation treatments were analyzed separately. Within each group and metric, we analyzed all salamanders ("Overall”), and then separately analyzed striped, unstriped, male, and female salamanders. $N$ is the number of individuals in the analysis. 95\% confidence intervals were inferred from 1000 parametric bootstraps. $P$-values represent significance of likelihood ratio tests, and $P$-values and their corresponding $R$ values that were significant after correction for multiple comparisons are shown in bold. Results for the proportion of flies eaten are presented on both the link scale and original scale, but $P$-values and interpretations were based upon the link scale only.

is caused not only by the delay in feeding but also a reduced rate by which individuals feed, as the difference between estimated control and predation latencies (17.1 sec) does not fully account for the difference in number of flies eaten in $10 \mathrm{~min}$. Calculating the number of flies eaten per minute after subtracting each group's estimated latency period, the rate of feeding for the predation group is only $1.14 \mathrm{flies} / \mathrm{min}$ compared to 
Table 2.

Repeatability values for proportion of flies eaten, using arcsine-transformed data and a Gaussian GLMM to extract variance components.

\begin{tabular}{|c|c|c|c|c|}
\hline \multicolumn{5}{|c|}{ Arcsine-transformed proportion } \\
\hline \multicolumn{5}{|c|}{ Flies eaten: Control } \\
\hline & $N$ & $R$ & $95 \% \mathrm{CI}$ & $P$ \\
\hline Overall & 40 & 0.43 & $0.22-0.60$ & 0.000 \\
\hline Striped & 20 & 0.44 & $0.13-0.69$ & 0.001 \\
\hline Unstriped & 20 & 0.44 & $0.10-0.67$ & 0.002 \\
\hline Male & 20 & 0.47 & $0.15-0.70$ & 0.001 \\
\hline Female & 20 & 0.41 & $0.10-0.65$ & 0.003 \\
\hline \multicolumn{5}{|c|}{ Flies eaten: Predation } \\
\hline Overall & 40 & 0.42 & $0.21-0.60$ & 0.000 \\
\hline Striped & 20 & 0.36 & $0.03-0.61$ & 0.009 \\
\hline Unstriped & 20 & 0.45 & $0.15-0.70$ & 0.001 \\
\hline Male & 20 & 0.31 & $0.01-0.56$ & 0.025 \\
\hline Female & 20 & 0.48 & $0.17-0.71$ & 0.000 \\
\hline
\end{tabular}

the control rate of 1.29 flies/min. The number of prey eaten thus provides unique information pertaining to the rate of feeding after the latency period ends. Furthermore, our repeatability analyses show that the foraging rate is consistently repeatable following simulated predation, whereas repeatability of latency varies across morphs and sexes. Based on our observations while conducting trials, latency to first feed appears to be influenced in part by the random chance that a fly moved close enough to the salamander to stimulate foraging. The rate of foraging thereafter provides an additional metric of the sustained effect of the predation attempt that is more consistent within individuals. Because the rate of consumption was repeatable for both control and attacked salamanders, it is can be considered a reliable foraging metric for studies of behavioral syndromes in similar species. We note that we analyzed our data as the proportion of flies eaten out of those provided using a binomial GLMM, and we obtained significant, but substantially lower repeatability estimates compared to the widely used arcsine transformation. Although our interpretations of which groups would exhibit repeatable behavior would not have changed using the arcsine transformation, future studies should be aware that proportion data analyzed with a binomial GLMM, which has advantages over the transformation of proportion or count data (Nakagawa \& Schielzeth 2010), may yield considerably lower repeatability estimates. Considering that laboratory studies may provide lower repeatability estimates than field studies (Bell et al. 2009), higher repeatability estimates could be obtained through measurements in the field. 
Similarity between males and females in their degree of bold behavior (i.e. mean latency to feed and feeding rate) following simulated predation is consistent with studies showing that both sexes exhibit territorial and aggressive behavior (Jaeger 1984; Horne 1988; Mathis 1991; Reiter et al. 2014), and boldness and aggressiveness tend to be correlated (Sih et al. 2004). The lack of a difference between color morphs in mean latencies or feeding rates following simulated predation, however, is somewhat surprising given previous studies of polymorphic populations. Relative to the unstriped morph, striped salamanders have been shown to secure territories more often, behave more aggressively towards intruders (Reiter et al. 2014), and spend a longer time immobile and in an aggressive posture when exposed to a snake chemical cue (Venesky \& Anthony 2007). We had therefore hypothesized that the striped morph would be bolder following a predation attempt and resume foraging sooner. Perhaps immobility or aggressive postures may not translate into differences in foraging behavior following the stimulus, or the greater severity of our simulated predation attempt (physically grasping the salamander) reduces the disparity between the striped and unstriped morphs. We measured only foraging behavior, and measuring aggressive displays, fleeing behavior, or immobility may provide additional insights relevant to bold-aggressive syndromes across color morphs (Sih et al. 2004).

Our most surprising finding is that latency to feed following simulated predation was repeatable for unstriped and male salamanders, but not for striped and female salamanders, and not for any subset of control salamanders. In another study that incorporated a simulated predation attempt to assess boldness, juvenile Spotted salamanders (Ambystoma maculatum) did not show repeatable behavior in their subsequent movement or use of a refuge (Koenig \& Ousterhout 2018). Our findings suggest first that latency to engage in some activity (e.g. foraging) may be distinct from latency to move, and that sexes or morphs in a polymorphic system can differ in the repeatability of their responses. Second, while color morphs and sexes in $P$. cinereus are known to vary in certain traits in studied populations, the repeatability of these traits can differ in ways that are relevant to the study of behavioral syndromes. For example, the propensity to disperse is often thought to be a trait of a bold personality (Fraser et al. 2001), and in one monomorphic population of $P$. cinereus, males were found to disperse further from their natal site than females (Liebgold et al. 2011). In a subsequent study of a polymorphic population, unstriped salamanders, but neither sex, were found to disperse further (Grant \& Liebgold 2017). The presence of sexbiased dispersal in a monomorphic population, while lacking in a polymorphic population, suggests that repeatability of bold behavior in unstriped salamanders and in male salamanders could be the result of distinct processes: males may evolve increased and more repeatable dispersal ability as a part of a bold personality, at least in part, to avoid inbreeding. Unstriped salamanders, on the other hand, may evolve increased and more repeatable dispersal behavior as a consequence of their lower rates of territoriality and higher frequency of encounters with predators relative to the striped morph (Grant \& Liebgold 2017). If this is true, the relationship between boldness and aggressiveness for color morphs in $P$. cinereus must be considered carefully. Both field and museum surveys have shown that the unstriped morph has higher rates of autotomized tails, indicative of more frequent encounters with predators or higher survival of these encounters (Moreno 1989; Venesky \& Anthony 2007). The unstriped morph may be consistently bolder while foraging in the face of predation risk as it moves through the leaf litter matrix, but the striped morph may act more aggressively in territorial interactions. 
Maintenance of color polymorphism, or the fixation of either morph, could be related to the spatial and temporal variation across populations. Predation risk and prey abundance, for example, likely vary considerably across the large geographic range of $P$. cinereus, as well as between years because temperature and moisture greatly affect individuals' ability to emerge and feed on small invertebrates (Heatwole 1962). Threespined sticklebacks (Gasterosteus aculeatus), like red-backed salamanders, exhibit territorial behavior (Peeke \& Veno 1976), and in populations with strong predation pressure, individuals that are bolder in encounters with predators are also more aggressive towards conspecifics. However, this correlation was not present in populations with relaxed predation (Dingemanse et al. 2007). Furthermore, prey abundance can influence the fitness of some trait combinations. In invasive cane toads (Rhinella marina), behavioral types range from "asocial-bold" to "social-shy". When comparing which individual captured a lone prey item in competition trials, the asocial-bold individual won significantly more often. However, when the pair of toads was presented with multiple crickets, the social-shy individual captured significantly more prey items (González-Bernal et al. 2014). We did not find that one morph of $P$. cinereus was necessarily bolder, but rather that the unstriped morph had a more repeatable response to a simulated predation attempt. The lower rates of territorial acquisition of the unstriped morph from this population may demand that they consistently take greater risk while foraging to acquire adequate resources. Indeed, the unstriped morph in our source population has been shown to have a diet of less profitable prey in the fall, possibly due to territories associated with high-quality prey being dominated by striped salamanders (Anthony et al. 2008, 2017; Hantak et al. 2020). Dietary differences at a polymorphic site are notable because in a comparison of multiple monomorphic and polymorphic populations, Hantak et al. (2020) found that diets of salamanders from monomorphic populations had the same dietary breadth as their respective morph in polymorphic populations. These results suggested that both morphs are generalists and would consume similar resources at our study site if not for competition with the other morph. In addition, the greater instances of tail breakage (Moreno 1989; Venesky \& Anthony 2007) and higher hematological stress indices of the unstriped morph (Davis \& Milanovich 2010) support the notion that the unstriped morph encounters predators more frequently. The repeatability of the unstriped morph's latency to feed following predation attempts may be driven by a more consistent lifetime risk of encountering predators in the leaf litter, whereas the striped morph may show more behavioral variation as they are more likely to acquire territories upon reaching adulthood.

Behaviors within a polymorphic population may vary across age classes, sexes, or morphs. We have shown that consistency of individual behavior itself can vary across sex and color morph, and that repeatability varies across contexts (i.e. with or without the threat of predation) and behavioral metrics. For studies of behavioral syndromes, differences in repeatability across groups can have meaningful implications if one sex or morph tends to exhibit more consistent behavior over time or across contexts, even if groups differ little on average for a behavior. Particularly for polymorphic species, such differences in repeatability may correspond to alternative ecological strategies. Polymorphic species, including $P$. cinereus, often exhibit variation in morph frequencies across populations (Cosentino et al. 2017), and comparison of behavioral repeatability and syndromes across monomorphic and polymorphic populations offers an exciting future direction of research. 


\section{FUNDING}

This project was supported by internal research funding from John Carroll University. C.D. Anthony was supported by a George Grauel Faculty Fellowship.

\section{ETHICAL STANDARD}

Salamanders were not returned to the field because of the risk of laboratory-borne disease transmission. After the current experiment, they were used in pilot studies examining effects of Batrachochytrium dendrobatidis. Salamanders were eventually euthanized following standard humane procedures (Simmons 2015) and deposited in the Cleveland Museum of Natural History.

\section{AUTHOR CONTRIBUTIONS}

All authors planned the experiment. B.P. Waldron and M.C. Ganzfried conducted behavioral trials, and performed statistics. B.P. Waldron wrote the manuscript, and M.C. Ganzfried, C. D. Anthony and C.-A.M. Hickerson reviewed the drafts.

\section{REFERENCES}

Acord MA, Anthony CD, Hickerson CAM. 2013. Assortative mating in a polymorphic salamander. Copeia. (4):676-683. doi:10.1643/CE-13-003

Anthony CD, Jaworski K, Messner M, Hickerson CAM. 2017. Differences in prey availability within the territories of striped and unstriped Eastern Red-backed Salamanders (Plethodon cinereus). Herpetol Rev. 48:509-514.

Anthony CD, Pfingsten RA. 2013. Eastern Red-backed Salamander. Plethodon cinereus (Green 1818). In: Pfingsten RA, editor. Amphibians of Ohio. Columbus (OH): Ohio Biological Survey, Inc.; p. 335-360.

Anthony CD, Venesky MD, Hickerson CAM. 2008. Ecological separation in a polymorphic terrestrial salamander. J Anim Ecol. 77(4):646-653. doi:10.1111/j.1365-2656.2008.01398.x

Ballew NG, Mittelbach GG, Scribner KT. 2017. Fitness consequences of boldness in juvenile and adult largemouth bass. Am Nat. 189(4):396-406. doi:10.1086/690909

Bates D, Mächler M, Bolker B, Walker S. 2015. Fitting linear mixed-effects models using lme4. J Stat Softw. 67(1):1-48. doi:10.18637/jss.v067.i01 
Bell AM, Hankison SJ, Laskowski KL. 2009. The repeatability of behaviour: a meta-analysis. Anim Behav. 77(4):771-783. doi:10.1016/j.anbehav.2008.12.022

Brodie ED, Russell NH. 1999. The consistency of individual differences in behaviour: temperature effects on antipredator behaviour in garter snakes. Anim Behav. 57(2):445-451. doi:10.1006/anbe.1998.0990

Cosentino BJ, Droney DC. 2016. Movement behaviour of woodland salamanders is repeatable and varies with forest age in a fragmented landscape. Anim Behav. 121:137-146. doi:10.1016/j.anbehav.2016.08.013

Cosentino BJ, Moore JD, Karraker NE, Ouellet M, Gibbs JP. 2017. Evolutionary response to global change: climate and land use interact to shape color polymorphism in a woodland salamander. Ecol Evol. 7(14):5426-5434. doi:10.1002/ece3.3118

Davis AK, Milanovich J. 2010. Lead-phase and red-stripe color morphs of Red-backed salamanders (Plethodon cinereus) differ in hematological stress indices: a consequence of differential predation pressure? Curr Zool. 56(2):238-243. doi:10.1093/czoolo/56.2.238

Dingemanse NJ, Wright J, Kazem AJ, Thomas DK, Hickling R, Dawnay N. 2007. Behavioural syndromes differ predictably between 12 populations of three-spined stickleback. J Anim Ecol. 76(6):1128-1138. doi:10.1111/j.1365-2656.2007.01284.x

Ehlman SM, Halpin R, Jones C, Munson A, Pollack L, Sih A. 2019. Intermediate turbidity elicits the greatest antipredator response and generates repeatable behaviour in mosquitofish. Anim Behav. 158:101-108. doi:10.1016/j.anbehav.2019.10.006

Fraser DF, Gilliam JF, Daley MJ, Le AN, Skalski GT. 2001. Explaining leptokurtic movement distributions: intrapopulation variation in boldness and exploration. Am Nat. 158 (2):124-135. doi:10.1086/321307

Gibbons ME, Ferguson AM, Lee DR. 2005. Both learning and heritability affect foraging behaviour of red-backed salamanders, Plethodon cinereus. Anim Behav. 69(3):721-732. doi:10.1016/j.anbehav.2004.06.021

González-Bernal E, Brown GP, Shine R, Auge H. 2014. Invasive cane toads: social facilitation depends upon an individual's personality. PLoS ONE. 9(7):e102880. doi:10.1371/journal. pone. 0102880

Grant AH, Liebgold EB. 2017. Color-biased dispersal inferred by fine-scale genetic spatial autocorrelation in a color polymorphic salamander. J Hered. 108(5):588-593. doi:10.1093/jhered/esx040

Hantak MM, Brooks KM, Hickerson CAM, Anthony CD, Kuchta SR. 2020. A spatiotemporal assessment of dietary partitioning between color morphs of a terrestrial salamander. Copeia. 108(4):727-736. doi:10.1643/CE-19-264

Heatwole H. 1962. Environmental factors influencing local distribution and activity of the salamander, Plethodon cinereus. Ecology. 43(3):460-472. doi:10.2307/1933374

Horne EA. 1988. Aggressive behavior of female red-backed salamanders. Herpetologica. 44 (2):203-209.

Jaeger RG. 1984. Agonistic behavior of the red-backed salamander. Copeia. (2):309-314. doi:10.2307/1445186

Jaeger RG, Gollman B, Anthony CD, Gabor CR, Kohn N. 2016. Behavioral ecology of the Eastern Red-backed salamander: 50 years of research. Oxford (UK): Oxford University Press.

Jenkins SH. 2011. Sex differences in repeatability of food-hoarding behaviour of kangaroo rats. Anim Behav. 81(6):1155-1162. doi:10.1016/j.anbehav.2011.02.021

Kelleher SR, Silla AJ, Byrne PG. 2018. Animal personality and behavioral syndromes in amphibians: a review of the evidence, experimental approaches, and implications for conservation. Behav Ecol Sociobiol. 72(5):79. doi:10.1007/s00265-018-2493-7

Koenig AM, Ousterhout BH. 2018. Behavioral syndrome persists over metamorphosis in a pond-breeding amphibian. Behav Ecol Sociobiol. 72(12):1-12. doi:10.1007/s00265-0182595-2 
Lenth R, Singmann H, Love J, Buerkner P, Herve M 2019. emmeans: estimated marginal means, aka least-squares means (version 1.3. 4). Available from: https:/cran.r-project.org/ web/packages/emmeans/emmeans.pdf [Accessed 2021 May 5].

Liebgold EB, Brodie ED, Cabe PR. 2011. Female philopatry and male-biased dispersal in a direct-developing salamander, Plethodon cinereus. Mol Ecol. 20(2):249-257. doi:10.1111/ j.1365-294X.2010.04946.X

Mathis A. 1991. Territories of male and female terrestrial salamanders: costs, benefits, and intersexual spatial associations. Oecologia. 86(3):433-440. doi:10.1007/BF00317613

Mazza V, Jacob J, Dammhahn M, Zaccaroni M, Eccard JA. 2019. Individual variation in cognitive style reflects foraging and anti-predator strategies in a small mammal. Sci Rep. 9 (1):1-9. doi:10.1038/s41598-019-46582-1

Moreno G. 1989. Behavioral and physiological differentiation between the color morphs of the salamander, Plethodon cinereus. J Herpetol. 23(4):335-341. doi:10.2307/1564043

Nakagawa S, Schielzeth H. 2010. Repeatability for Gaussian and non-Gaussian data: a practical guide for biologists. Biol Rev. 85(4):935-956. doi:10.1111/j.1469-185X.2010.00141.x

Paluh DJ, Eddy C, Ivanov K, Hickerson CAM, Anthony CD. 2015. Selective foraging on ants by a terrestrial polymorphic salamander. Am Midl Nat. 174(2):265-277. doi:10.1674/00030031-174.2.265

Peeke HVS, Veno A. 1976. Response independent habituation of territorial aggression in the Three-spined Stickleback (Gasterosteus aculeatus). Z Tierpsychol. 40(1):53-58. doi:10.1111/ j.1439-0310.1976.tb00925.x

R Core Team. 2019. R: A language and environment for statistical computing. Version 3.5.3. Vienna (Austria): R Foundation for Statistical Computing.

Réale D, Reader SM, Sol D, McDougall PT, Dingemanse NJ. 2007. Integrating animal temperament within ecology and evolution. Biol Rev. 82(2):291-318. doi:10.1111/j.1469185X.2007.00010.x

Reiter MK, Anthony CD, Hickerson CAM. 2014. Territorial behavior and ecological divergence in a polymorphic salamander. Copeia. (3):481-488. doi:10.1643/CE-13-154

Roy T, Shukla R, Bhat A. 2017. Risk-taking during feeding: between-and within-population variation and repeatability across contexts among wild zebrafish. Zebrafish. 14 (5):393-403. doi:10.1089/zeb.2017.1442

Schuett W, Dall SR. 2009. Sex differences, social context and personality in zebra finches, Taeniopygia guttata. Anim Behav. 77(5):1041-1050. doi:10.1016/j.anbehav.2008.12.024

Schuett W, Tregenza T, Dall SR. 2010. Sexual selection and animal personality. Biol Rev. 85 (2):217-246. doi:10.1111/j.1469-185X.2009.00101.x

Sih A, Bell A, Johnson JC. 2004. Behavioral syndromes: an ecological and evolutionary overview. Trends Ecol Evol. 19(7):372-378. doi:10.1016/j.tree.2004.04.009

Sih A, Cote J, Evans M, Fogarty S, Pruitt J. 2012. Ecological implications of behavioural syndromes. Ecol Lett. 15(3):278-289. doi:10.1111/j.1461-0248.2011.01731.x

Simmons JE. 2015. Herpetological collecting and collections management. 3rd ed. Salt Lake City (UT): Society for the Study of Amphibians and Reptiles.

Smith BR, Blumstein DT. 2008. Fitness consequences of personality: a meta-analysis. Behav Ecol. 19(2):448-455. doi:10.1093/beheco/arm144

Sokal RR, Rohlf FJ. 1981. Biometry: the principles and practice of statistics in biological research. New York (NY): W.H. Freeman \& Co.

Stoffel MA, Nakagawa S, Schielzeth H, Goslee S. 2017. rptR: repeatability estimation and variance decomposition by generalized linear mixed-effects models. Meth Ecol Evol. 8 (11):1639-1644. doi:10.1111/2041-210X.12797

Strickland K, Frère CH, Taborsky M. 2018. Predictable males and unpredictable females: repeatability of sociability in eastern water dragons. Behav Ecol. 29(1):236-243. doi:10.1093/beheco/arx148

Venesky MD, Anthony CD. 2007. Antipredator adaptations and predator avoidance by two color morphs of the eastern red-backed salamander, Plethodon cinereus. Herpetologica. 63 (4):450-458. doi:10.1655/0018-0831(2007)63[450:AAAPAB]2.0.CO;2 